Tiere erwähnt werden, nämlich der bei dem Kaninchen beobachtete Eintritt der Ovulation erst nach erfolgter Kopulation. Aber gerade diese analoge Tatsache, die auch nach Begattung mit einem sterilen $\hat{f}$ oder nach elektrischer Reizung ohne jegliche Begattung in Erscheinung treten kann, läßt vorerst die Möglichkeit offen, daß in unserem Falle der die Eiablage befördernde Kopulationseinfluß auch auf ein nervöses Phänomen zurückzuführen wäre, sei es in unmittelbarer Auswirkung des Kopulationsvorganges oder eher noch - da die Eiablage erst eine gewisse Zeit nach der Kopulation einsetzt - als reflektorische Folge des Füllungszustandes oder der entleerenden Bewegungen von Bursa copulatrix oder Receptaculum seminis. Die vorgesehenen Untersuchungen werden auch hierin vermutlich weitere Aufklärung geben.

\title{
Spontane Mutabilität bei Bacterium prodigiosum
}

\author{
Von Reinhard Kaplan \\ Aus dem Kaiser-Wilhelm-Institut für Züchtungsforschung, E r w in - B a u r - Institut, Voldagsen \\ (7. Naturforschg. 2 b, 308-312 [1947]; eingegangen am 19. März 1947)
}

\begin{abstract}
Die biophysikalische Analyse des Mutationsprozesses ergibt eine Charakterisierung der Gene in Hinsicht auf ihre stoffliche Natur, während die Bastardanalyse lediglich die Lokalisation dieser ihr im Wesen unbekannt bleibenden Erbfaktoren gestattet. Darum kann jene Methode auch bei nichtkreuzbaren Organismen eine Entscheidung darüber herbeiführen, ob die für Erbvariationen verantwortlichen Faktoren dieselbe stoffliche Natur besitzen wie die Gene der höheren sexuellen Organismen. Die spontanen Erbvariationen von Bact. prodigiosum, die ein Umschlagen der Koloniefärbung erzeugen, erwiesen sich als nach der Eintrefferfunktion von der Lagerzeit der Zellen abhängig und ergaben Temperaturquotienten, denen Aktivierungsenergien von etwa $0,6 \mathrm{eV}$ entsprechen. Daraus und aus den Absolutgrößen der Mutationsraten ließ sich weiterhin eine anscheinend einfache Beziehung zwischen Aktivierungsenergie und -entropie finden, die auch Vermutungen über die Größenordnung der beteiligten Genzahl gestattete. Danach scheint der untersuchte Variationsprozeß durch relativ wenige Erbfaktoren (vielleicht nur einen) zustandezukommen, die den mutablen Genen höherer Organismen entsprechen.
\end{abstract}

$\mathrm{D}^{\mathrm{i}}$ e Aufklärung des Mutationsprozesses bei höheren Organismen hat uns Möglichkeiten geliefert, die Gene direkt durch ihr stoffliches Wesen (ablesbar an den Besonderheiten der $\mathrm{Mu}$ tationsentstehung) $\mathrm{zu}$ charakterisieren, ohne dabei die Lokalisation in den Chromosomen (erschließbar aus dem Erbgang nach Kreuzung) zu. berücksichtigen. Sehen wir diese Lage der Gene im Kern als weniger wesentlich an als ihre spezielle molekulare Struktur, so gibt uns die systematische quantitative Analyse erblicher Variationen eine Handhabe, die Frage nach dem Vorhandensein von Genen auch für solche Organismen zu beantworten, die keine Sexualität (Kopulation und Meiose) besitzen. Für die Bakterien sind schon viele Tatsachen über Erbvariationen bekannt, die es aussichtsreich erscheinen lassen, mit den Methoden der Mutationsforschung diese Frage anzugehen. Dies erscheint wichtig, weil sie zum Teil Möglichkeiten der weiteren Aufklärung des Genbaues bieten könnten, die bei den höhe- ren Organismen nicht vorhanden sind. Eine Sichtung der Literatur ergab, daß sprunghafte Erbvariationen der Bakterien („Dissoziation“ usw.) durch die gleichen Agentien erhalten wurden, die auch bei höheren Organismen Mutationen erzeugen (Alterung, Strahlen, Chemikalien). Unter den wenigen quantitativen Ergebnissen erscheint am wichtigsten der Nachweis exponentieller Wirkung der Röntgenstrahlendosis auf die Variantenrate ${ }^{1 .}$ Dies zeigt, daß zumindest gewisse Varianten sehr wohl echte Genmutationen sein können. Die Ergebnisse über die Strahlentötung der Bakterien hatten schon seit längerem ihre Erklärung durch Annahme letalmutationsähnlicher Vorgänge nahegelegt ${ }^{2}$. Die Lage und Zusammenfassung der betreffenden Gene in Chromosomen bzw. Kernen ist natürlich für diese Fragestellung von sekundärer Bedeutung. Die Bakteriengene brauchen u. L. weder in Chromosomen aggregiert zu sein, noch

1 Ralph E. Lincoln, Genetics 25, 125 [1940].

2 P. Jordan, Protoplasma 32, 464 [1939]. 
evtl. diese in echten Kernen (mit Membran, Spindelmechanismus, Nukleolen usw.) zu liegen.

Zur Aufhellung des bakteriellen Erbvariationsmechanismus ist eine Methode nötig, die an einer großen Anzahl von Einzelzellen die Häufigkeit von Erbvariationen, die in ihnen entstanden sind und sich an den daraus hervorgehenden Nachkommenschaften (Einzelkolonien) manifestieren, festzustellen gestattet. Denn die quantitative Abhängigkeit des Prozentsatzes der mutierten unter einer großen Zahl geprüfter Zellen, der Mutationsrate, von den einwirkenden Agentien ist es ja, was uns erst die intimen Züge des Mutationsprozesses und des Gens (Monomolekularität; Größe des Strahlentreffbereichs, Aktivierungsenergie usw.) erkennen läßt. Die einfachste Methode besteht darin, nach Einwirkung des Agens auf die Zellen eine Suspension von möglichst hohem Prozentsatz an Einzelzellen, z. B. durch Schütteln, herzustellen und von dieser kleine Mengen zur „Aussaat" auf Agarplatten breitzustreichen. Praktisch jede der daraus hervorgehenden Kolonien stellt darn die Nachkommenschaft einer Zelle der Suspension dar und kann deren erbliche Konstitution anzeigen. Als ein hierzu (bezüglich Einzelligkeit u. a. Eigenschaften) geeigneter Organismus erwies sich Bacterium prodigiosum (= Serratia marcescens). Da bei ihm das spontane Umschlagen eines Bruchteils der Zellen zu abgeänderten Kolonien (z. B. rot nach weiß und umgekehrt) häufig vorkommt und die Erblichkeit dieser Variation nachgewiesen ist ${ }^{3}$, lag es nahe, diese spontanen Änderungen mit den bei höheren Organismen bekannten spontanen Mutationen zu vergleichen. Über ein erstes Ergebnis dieses Vergleichs sei hier berichtet.

Die Versuchsmethode bestand darin, aus einer Einzelkolonie einer Petrischalenkultur (,Stammplatte“) eine Zellensuspension herzustellen, von der dann eine Impföse voll auf eine weitere Petrischale mit synthetischem Nähragar (Glycerin - Zitronensäure - Ammonphosphat, $p_{\mathrm{H}} 7,0$ ) ausgespatelt wurde. Die so erhaltene ,Zählplatte“ wurde bei $30^{\circ} \mathrm{C}$ bebrütet und die angewachsenen roten $(r)$ und weißen $(w)$ Kolonien (insgesamt meist mehrere hundert je Platte) ausgezählt. Das Verhältnis der $r$-bzw. $w$-Kolonien zur Gesamtzahl der geprüften entspricht dann der Mutationsrate der Zellen der Stammkolonie. Als von mutierten Zellen abstammend wurden nur die total umgeschlagenen Kolonien gezählt, solche mit Sektoren dagegen als unmutiert gewertet, da die sektorerzeugende Mutation erst im Verlauf der Kolonieentwicklung entstanden sein mußte.

3 M. I. Bunting, J. Bacteriology 40, 57 [1940].

\begin{tabular}{|c|c|}
\hline $\begin{array}{c}\text { Kolonienfläche } \\
\left(\mathrm{mm}^{2}\right)\end{array}$ & Mutationsrate \\
\hline 0,27 & $9 / 43=20,9^{\circ} \%$ \\
0,27 & $34 / 180=18,9 "$ \\
0,35 & $26 / 146=17,8 \%$ \\
0,49 & $12 / 52=23,1 \%$ \\
0,99 & $71 / 361=19,6 \%$ \\
1,22 & $52 / 272=19,1 \%$ \\
1,40 & $43 / 190=22,6 "$ \\
2,17 & $71 / 432=16,5 \%$ \\
2,85 & $77 / 419=18,4 \%$ \\
\hline Durchschnitt: & $395 / 2095=18,9 \%$ \\
$\chi^{2} 8=12,0$ & $P_{\text {Hom. }}=0,15 \%$ \\
\hline
\end{tabular}

Tab. Mutationsraten von $9 r$-Kolonien gleichen Alters (10 Tage bei $30^{\circ} \mathrm{C}$ ), aber verschiedener 'Zellzahl (Fläche).

Als erste Frage wurde geprüft, ob das Umschlagen der Koloniefarbe, z. B. von $r$ nach $w$, durch den Ablauf der Zellteilung induziert wird (durch zufällige Entmischung von frei im Plasma liegenden Erbpartikeln o. ä.) oder unabhängig von dieser geschieht. Sie wurde entschieden durch Feststellung der Mutationsrate in gleich alten, aber verschieden großen Kolonien einer Stammplatte, bei denen also im gleichen Zeitraum verschieden viele Zellteilungen, wohl durch zufällige Nährstoffunterschiede, stattgefunden hatten. Wie die Tabelle zeigt, ist trotz eines Unterschiedes des Flächeninhaltes der Kolonien von etwa $1: 10$. dem ein wahrscheinlich noch größerer Zellzahlenunterschied entspricht, kein Unterschied der $\mathrm{Mu}^{-}$ tationsrate nachweisbar. Hierdurch wird für die geprüfte Zeitspanne eine weitgehende Unabhängigkeit des Erbvariationsvorgangs von der Zellteilung angezeigt, wie wir es von den Genmutationen höherer Organismen gewöhnt sind.

Das zweite angeschnittene Problem war die Abhängigkeit der Mutationsrate von der Zeitdauer, während der die Mutationen stattfinden können. Von den Mutationen höherer Organismen wissen wir, daß sie Umlagerungen molekülartiger Atomverbände durch einzelne überschwellige Wärmeschwingungen, analog den monomolekularen chemischen Reaktionen, sind. Für derartige Prozesse ist eine exponentielle Zeitabhängigkeit typisch, falls diese Funktion nicht von sekundären Faktoren verformt wird. Zur Feststellung der Abhängigkeit der Mutationsrate von der Zeit wurde die Beobachtung ausgenutzt, daß die Kolonien nach etwa zwei Tagen ihr Wachstum wegen Nährstoffmangels einstellen und die Zellen sich 
nach Eintreten dieses Ruhestadiums noch eine Zeitlang lebend und wahrscheinlich ungeteilt halten. Es wurden also $r$ - und $w$-Stammplatten (insgesamt $28 r, 22 w$ ) im Brutschrank weiter aufbewahrt und von ihnen nach gewissen Zeiten aus Einzelkolonien Zählplatten abgeimpft. Durch Auszählung dieser wurden die Mutationsraten in den Kolonien der Stammplatte erhalten. Ein Teil der Zählplatten wurde als Stammplatten für weitere gleichartige Versuche verwendet. Die Ergebnisse der Abimpfungen von $r$-Kolonien aus den Stammplatten ist in Abb.1, die von $w$-Kolonien in Abb. 2

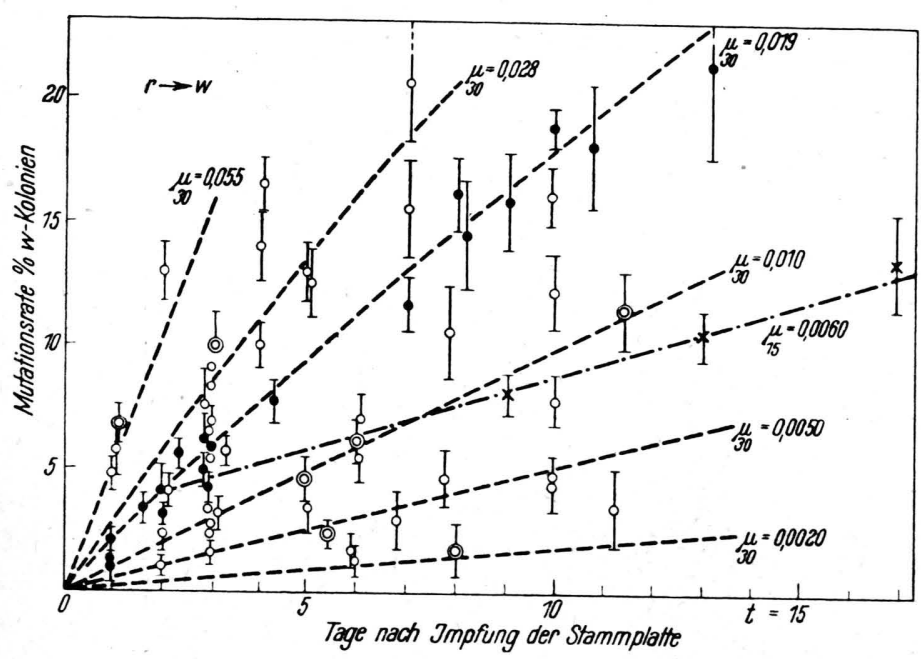

Abb. 1. Abhängigkeit der Mutationsrate $r \rightarrow w$ ( $\%$ weiße Kolonien) vom Alter roter Stammkolonien.

dargestellt. Die eingezeichneten Fehlerspannen der Prozentsätze sind nach der Näherungsformel $\sqrt{p \cdot q / n}$ berechnet ( $p=\%$ mutierte, $q=\%$ unmutierte Kolonien, $n=$ Gesamtzahl ausgewerteter Kolonien der Platte).

Die Versuche mit $r$-Kolonien ergaben zunächst eine deutliche Zeitproportionalität (Abb. 1: volle Kurvenpunkte $\bullet, \mu_{30}=0,019$ ), die dem unteren Teil einer Exponentialfunktion entspricht. Das bei längerer Aufbewahrung stattfindende Austrocknen und Absterben der Kolonien begrenzte die Versuchsdauer. Im Verlaufe der Versuche, bei denen die Mutationsraten von $57 r$-Kolonien (aus 10 Stammplatten) verschiedenen Alters festgestellt wurden (in Abb. 1 sind in einigen Kurvenpunkten die nahe beieinander liegenden Raten mehrerer Kolonien zusammengezogen), fanden sich auch insgesamt 7 Kolonien (Doppelkreise o ), die aus der gefundenen Zeitkurve herausfielen, also stabiler oder mutabler waren als der ,Normalstamm“. Wiederholte Versuche, sie als stabilere oder labilere Stämme zu isolieren, schlugen fehl, da ihre Nachkommenschaften (einfache Kreise o ) nicht konstant bezüglich der Zugehörigkeit zu einer „Mutabilitätslinie“ waren. Sie ergaben z.T. uneinheitliche Mutationsraten, die oft über oder unter den jeweiligen Elternstämmen lagen. Wie Abb. 1 zeigt, lassen sich diese „inkonstanten“ Kolonien etwa 5 Mutabilitätszeitfunktionen (---) zuordnen. Das beobachtete Entstehen dieser „Nebenlinięn“" aus der „Normallinie“ mit einer Häufigkeit von $7 / 57$ zeigt jedoch, daß der Unterschied beider Typen nur graduell ist und darin besteht, daß der Normalstamm häufiger Nachkommen seiner eigenen Mutabilität ergibt als die Nebenstämme.

Das unvorhergesehene "Springen“ der Mutationsrate zeigten auch analog den $r$-Nebenlinien alle untersuchten $w$-Kolonien bezüglich des Übergangs $w$ nach $r$. Ein relativ stabilerer „Normalstamm“ war hier nicht deutlich nachweisbar. Sehr wahrscheinlich bestehen aber auch hier Unterschiede in der „Intraphänmutabilität“ (s.u.) der einzelnen Linien, doch offenbaren sich diese nicht so deutlich wegen des kleinen Versuchsumfangs und wohl auch wegen ihrer geringeren reellen Größen. Die gefundenen Punkte ordnen sich etwa 4 Zeitlinien ein. Möglicherweise hätten sich bei weiterem Suchen in beiden Phänotypen $r$ und $w$ noch mehr solche Linien auffinden Iassen.

Da die Aufbewahrung der Stammplatten die Zellen allmählich absterben ließ, vielleicht aber auch nach Abschluß des Koloniewachstums noch Zellteilungen stattfinden, war zu prüfen, ob nicht die gefundene Zeitfunktion durch unterschiedliches Absterben oder Vermehren der $r$ - und $\boldsymbol{w}$ Zellen entstehen könnte. Bezeichnen wir mit $t$ die Zeit, $A_{0}$ das Ausgangsverhältnis der $r$ - und $\boldsymbol{w}$ Zellen und mit $\delta$ die Differenz der Sterbe- bzw. Wachstumsraten beider Zelltypen, so gilt unter Annahme des bei Bakterien weit verbreiteten exponentiellen Wachstums bzw. Absterbens für die Mutantenrate $m=1 / 1+A_{0} \cdot e^{ \pm \delta t} \approx 1 / 1+A_{0}(1 \pm \delta t)$. Die Zeitfunktion müßte dann also im Anfangsteil nach oben hyperbelähnlich konkav sein. Der gegenteilige experimentelle Kurvenverlauf zeigt demnach, daß Sterbe- oder Wachstumsvorgänge am Zustandekommen der gefundenen Zeitfunk- 
tion nicht wesentlich beteiligt sein können, sondern daß diese durch Ansammeln von Mutationen, die im Laufe der Zeit entstehen, erzeugt wird.

Wenn wir den beiden Phänotypen $r$ und $w$ nur je einen Genotyp zuordnen könnten, so müßte die Mutationsrate $m$ im Laufe der Zeit durch das Hinund Rückmutieren $(r \rightleftarrows w)$ einem Gleichgewichtszustand, analog einer reversiblen chemischen Reaktion, zustreben, der nur von den beiden Muta-

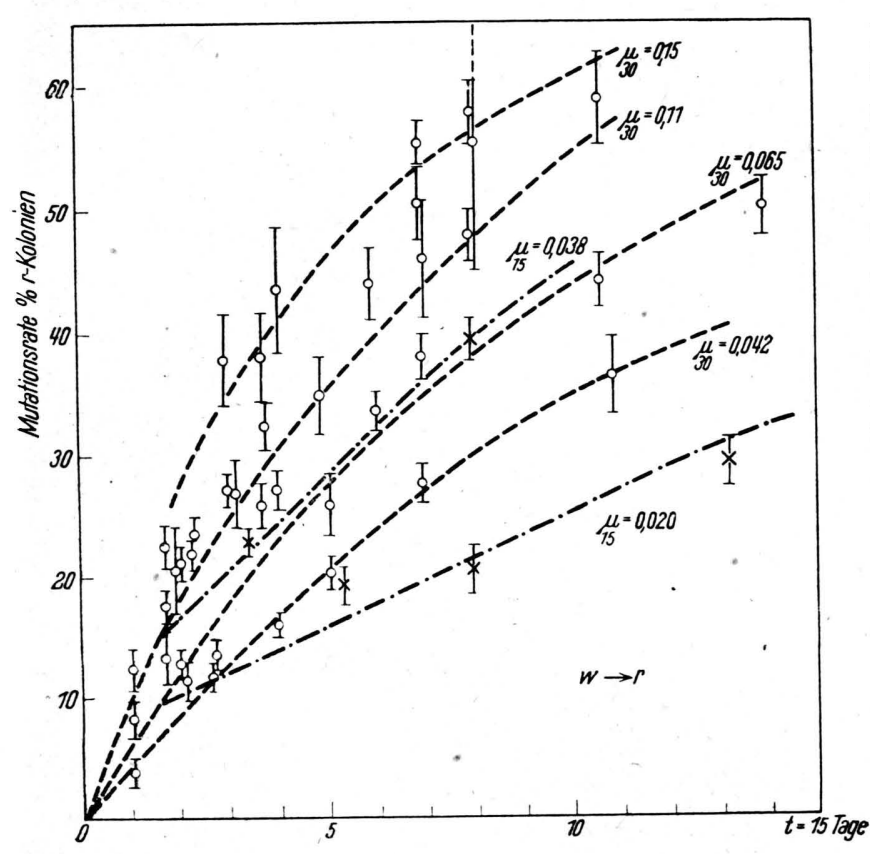

1bb. 2. Abhängigkeit der Mutationsrate $w \rightarrow r$ (\% rote Kolonien) vom Alter weißer Stammkolonien.

tionskonstanten $\mu_{r}$ und $\mu_{w}$ bestimmt würde: $m=m_{\infty}-\left(m_{\infty}-m_{0}\right) \cdot e-\left(\mu_{r}+\mu_{w}\right) t$, mit $m_{0}$ $=$ Ausgangsmutationsrate und $m_{\infty}=\mu_{r} / \mu_{r}+\mu_{w}$ $=$ Endmutationsrate. Setzen wir, was dem Fall der Versuche entspräche, $m_{0}=0$ und $e^{-x} \approx 1-x$. also $m \approx \mu_{r} \cdot t$, so ließen sich die Mutationskonstanten $\mu_{r}$ und $\mu_{w}$ aus dem'Anstieg der Kurven im Nullpunkt bestimmen. In unserem Falle müssen wir aber jeder gefundenen Zeitfunktion einen anderen Genotyp zuordnen, da ein bestimmter Genotyp (Gen, Allel) ja nicht nur durch sein von ihm erzeugtes Phän (hier also Koloniefarbe), sondern auch durch seine Mutabilität ckarakterisiert ist. Ferner hatten wir gesehen, daß zwischen den „Zeitlinien“ innerhalb eines Phäns mutative Übergänge stattfinden, die sich also nicht durch Umschlagen der Koloniefärbung, sondern nur durch Änderung der Mutationskonstante kundgeben. Wir wollen sie „Intraphänmutationen“" nennen. Das im Experiment zu findende Endgleichgewicht $m$ würde daher von einer sehr großen Zahl Reaktions-(= Mutations-)Konstanten abhängen, die im einzelnen kaum bestimmbar sind. Aus den gleichen Gründen entsprechen die den Kurven angeschriebenen Mutationskonstanten $\mu_{30}$ nur denjenigen Übergängen, die zu einem Umschlag der Koloniefärbung führen; die Intraphänmutationen kommen darin nicht zum Ausdruck. Die Frage, ob die gefundenen Genotypen mehreren verschiedenen Genen oder verschiedenen Allelen eines Gens entsprechen, läßt sich auf dem sonst üblichen Wege der Bastardanalyse mangels der Sexualität nicht entscheiden. Vorerst dürfen wir auf Grund der gefundenen Form der Zeitfunktion wohl mit ziemlicher Sicherheit annehmen, daß die untersuchten Erbvariationen des Bact. prodigiosum Prozesse von der gleichen Art darstellen, wie wir sie als Genmutationen von den höheren Organismen her kennen. Ihre relativ große spontane Häufigkeit im vorliegenden Falle, auch unter den Bakterien keine Normalerscheinung, läßt sie nicht den normal stabilen, sondern den mutablen Genen zuordnen.

Ein drittes Problem wurde durch die Abhängigkeit der Zeitfunktion von der Temperatur angeschnitten. Es wurden $r$ - und $\boldsymbol{w}$-Stammplatten aus den Linien ${ }^{(r)} \mu_{9.0}=0,019 \mathrm{bzw} .{ }^{(w)} \mu_{30}=0,11 \mathrm{zu}-$ nächst bei $30^{\circ} \mathrm{C} 2$ bzw. 1,5 Tage bebrütet, dann aber bei $15^{\circ} \mathrm{C}$ weitergehalten. Die tiefere Temperatur bewirkte eine Senkung der Mutationskonstanten $(X=$ empirische Punkte,...$---=$ Zeitfunktion), jedoch konnten bei dem $w$-Typ die zwei gefundenen $15^{\circ}$ - Linien wegen dessen häufigerer Intraphänmutationen nicht sicher bestimmten $30^{\circ}$ Linien zugeordnet werden. Am wahrscheinlichsten, auf Grund der bisher beobachteten Ubergänge, ist die Zuordnung von ${ }^{\left(w_{1}\right)} \mu_{15}=0,038 \mathrm{zu}$ ${ }^{\left(w_{1}\right)} \mu_{30}=0,11$ und ${ }^{\left(w_{2}\right)} \mu_{15}=0,020 \mathrm{zu}{ }^{(w)} \mu_{3.0}=0,065$.

Für die Gene von Drosophila ${ }^{4}$ konnte unter Anwendung des van 't $\mathrm{H}$ of f schen Gesetzes aus der Temperaturabhängigkeit der Mutabilität die Aktivierungsenergie $U=1,98 \cdot 10^{-4}\left(T_{1}^{-} \cdot T_{2} / T_{1}-T_{2}\right)$ $\lg \left(\mu_{1} / \mu_{2}\right) \mathrm{eV}$ errechnet werden, die eine Wärmeschwingung des Genmoleküls zur Entstehung einer Mutation überschreiten muß. Nach ${ }^{5}$ ergibt

${ }_{4}^{4} \mathrm{~N}$. W. Timofé eff-Ressovsky u. K. G. Z i m m e r, Z. Vererbungsl. 79, 530 [1941].

s K. Pätau u. N. W. Timón éeff-Ressovsky, Z. Vererbungsl. 81, 62 [1943]. 
sich für die $C l B$-Letalgene $1,35 \mathrm{eV}$, für ein relativ mutables $b b$-Allel $0,95 \mathrm{eV}$. Die gleiche Rechnung für unsere $r \rightarrow w$-Mutation mit ${ }^{(r)} \mu_{30}=0,019$ und ${ }^{(r)} \mu_{15}=0,0060$ Mut./Tag liefert $U_{r}=0,58 \mathrm{eV}$. Ordnet man den beiden $15^{\circ}-w$-Linien die beiden oben erwähnten $30^{\circ}$-Linien zu, so erhält man für diese Gene oder Allele $U_{w_{1}}=0,53 \mathrm{eV}$ und $U_{w_{2}}=0,59 \mathrm{eV}$. Die gefundenen niederen Aktivierungsenergien der Prodigiosum-Gene entsprechen also den Erwartungen, die man auf Grund der Befunde an den stabileren Drosophila-Genen haben kann.

Das Temperaturgesetz monomolekularer Reaktionen erlaubt eine noch etwas weitergehende Analyse der Befunde. Bei bekannter Aktivierungsenergie $U$ ergibt sich danach für die Mutationskonstante $\mu=n C \cdot e^{-U / k T}$; dabei ist $n$ die Gesamtzahl der Allele aller beteiligten Gene, $k=$ $0,86 \cdot 10^{-4} \mathrm{eV}$ und $C$ eine Zahl, die von der Molekülstruktur bestimmt ist und für normale Moleküle auf höchstens $5 \cdot 10^{17} \cdot \mathrm{Tag}^{-1}$ geschätzt wird ${ }^{4}$. Setzen wir die bekannten Werte für die $C l B$-Gene $\left(\mu_{20}=1,0 \cdot 10^{-4} \cdot \mathrm{Tag}^{-1}, U=1,35 \mathrm{eV}\right)$ ein, so erhalten wir $n C=2 \cdot 10^{19} \cdot \mathrm{Tag}^{-1}$. Die in Frage kommende Allelenzahl $n$ im $X$-Chromosom wird ${ }^{4}$ auf etwa $10^{4}$ geschätzt, so daß sich für $C$ etwa $10^{15}$ ergibt, was der erwähnten Schätzung nicht widersprechen würde. Für das besagte $b b$-Gen erhält man auf gleiche Weise $\left(\mu_{20}=6,5 \cdot 10^{-6}, U=0,95\right)$ für $n C=1,6 \cdot 10^{11}$. Da die Allelenzahl von $b b$ wahrscheinlich innerhalb einer Zehnerpotenz liegt, dürfte $C$ um $10^{11}$ betragen. Dies liegt aber trotz der großen Ungenauigkeit der Bestimmungen unter dem Wert der normalen $C l B$-Gene. Für unsere Prodigiosum-Mutationen erhalten wir $n C=8 \cdot 10^{7}(r), 6 \cdot 10^{7}\left(w_{1}\right)$ und $4 \cdot 10^{8}\left(w_{2}\right)$. Diese Werte liegen also wiederum unterhalb von $b b$. Tragen wir die Aktivierungsenergie $U$ gegen den der Aktivierungsentropie proportionalen Ausdruck $\log (n C)$ auf (siehe Abb.3), so scheinen sich die Werte aller behandelten Gene einer Geraden einzuordnen. Insbesondere liegen die Prodigiosum-Punkte etwa auf der Geraden durch die beiden für Einzelgene von Drosophila geltenden Punkte. Der für die ClB-,,Gensummen"-Muta- tionsraten geltende Punkt fällt dagegen deutlich heraus. Man kann somit vermuten, daß bei den untersuchten Prodigiosum-Mutationen zumindest nicht sehr viele Gene im Spiele sind, möglicherweise nur eines. Diese Vermutung wird gestützt durch demnächst zu veröffentlichende Ergebnisse von UV-Bestrahlungen: Eine Dosis, welche ein Überlebendenverhältnis von $10^{-5}$ ergibt, erzeugt eine ,Gensummen“-Mutationsrate verschiedenartiger Mutanten von $10 \%$, während die Mutations-

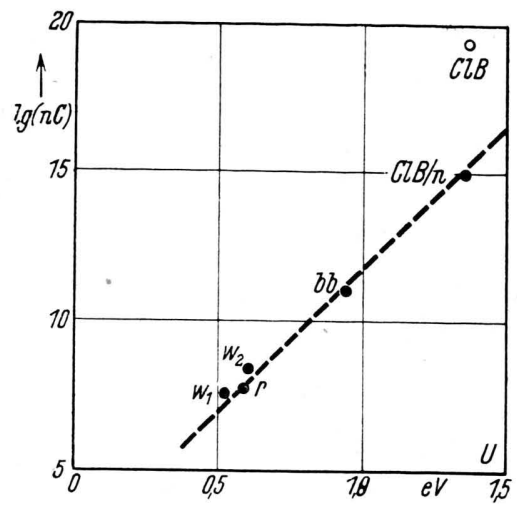

Abb. 3. Zusammenhang zwischen Aktivierungsenergie $(U)$ und -entropie $(\lg C)$ bei Genen von Drosophila melanogaster $(\mathrm{ClB}, \mathrm{bb})$ und Bacterium prodigiosum $\left(r, w_{1}, w_{2}\right)$.

rate $r \rightarrow w$ nicht nachweisbar erhöht ist und demnach unter 1\% liegt. Die Zahl der $r \rightarrow w$-Gene entspricht also höchstens 1/10 der an der Gensummenrate beteiligten Gene.

Wegen der großen Fehler in der Bestimmung der beiden Konstanten $U$ und $C$ erscheint es unsicher, daß der gefundene Zusammenhang real ist. Möglicherweise ist die Zahl der $C l B$-Gene wesentlich unterschätzt, sind die Temperaturkoeffizienten sehr ungenau bestimmt und die $\mu$ für Prodigiosum wegen der (nicht gezählten) Intraphänmutationen zu niedrig eingesetzt. Dadurch würden sich die $C$-Werte vielleicht denen von $b b$ nähern, wenn auch eine solche Verschiebung kaum eine Konstanz von $C$ gegenüber $U$ ergeben dürfte. 